\title{
STOCHASTIC REPRESENTATION OF A FRACTIONAL SUBDIFFUSION EQUATION. THE CASE OF INFINITELY DIVISIBLE WAITING TIMES, LÉVY NOISE AND SPACE-TIME-DEPENDENT COEFFICIENTS
}

\author{
MARCIN MAGDZIARZ AND TOMASZ ZORAWIK \\ (Communicated by Mark M. Meerschaert)
}

\begin{abstract}
In this paper we analyze a fractional Fokker-Planck equation describing subdiffusion in the general infinitely divisible (ID) setting. We show that in the case of space-time-dependent drift and diffusion and time-dependent jump coefficients, the corresponding stochastic process can be obtained by subordinating a two-dimensional system of Langevin equations driven by appropriate Brownian and Lévy noises. Our result solves the problem of stochastic representation of subdiffusive Fokker-Planck dynamics in full generality.
\end{abstract}

\section{Preliminaries}

Subdiffusion processes are characterized by the asymptotic power-law behavior of the variance $\operatorname{Var}(X(t)) \sim c t^{\alpha}$ as $t \rightarrow \infty$, where $0<\alpha<1$. A physical equation describing subdiffusion in the presence of space-dependent force $F(x)$ is the fractional Fokker-Planck equation (FFPE) 28 31 .

$$
\frac{\partial w(x, t)}{\partial t}={ }_{0} D_{t}^{1-\alpha}\left[-\frac{\partial}{\partial x} F(x)+\frac{\sigma^{2}}{2} \frac{\partial^{2}}{\partial x^{2}}\right] w(x, t),
$$

with the scale parameter $\sigma>0$ and the initial condition $w(x, 0)=\delta(x)$. Here

$$
{ }_{0} D_{t}^{1-\alpha} f(t)=\frac{1}{\Gamma(\alpha)} \frac{d}{d t} \int_{0}^{t}(t-s)^{\alpha-1} f(s) d s,
$$

$0<\alpha<1, f \in C^{1}([0, \infty))$, is the fractional derivative of the Riemann-Liouville type [36]. Equation (1.1) specifies probability density function (PDF) $w(x, t)$ of the subdiffusion process. It was derived in 29] in the framework of continuous-time random walk with heavy-tailed waiting times $[26,27$.

Stochastic process corresponding to (1.1) has the subordination form

$$
Y(t)=X\left(S_{\alpha}(t)\right), \quad t \geq 0,
$$

where $X$ is given by the following stochastic differential equation:

$$
d X(t)=F(X(t)) d t+\sigma d B(t), \quad X(0)=0,
$$

driven by Brownian motion $B$. Moreover, $S_{\alpha}$ is the inverse $\alpha$-stable subordinator

$$
S_{\alpha}(t)=\inf \left\{\tau>0: T_{\alpha}(\tau)>t\right\},
$$

Received by the editors September 3, 2014 and, in revised form, April 10, 2015. 2010 Mathematics Subject Classification. Primary 60G51; Secondary 60G22. 
which is assumed to be independent of $B$. Here, $T_{\alpha}$ is the $\alpha$-stable subordinator [10,38] with Laplace transform $\mathbb{E}\left[e^{-u T_{\alpha}(\tau)}\right]=e^{-\tau u^{\alpha}}, 0<\alpha<1$. As shown in [19, 20], PDF of $Y(t)=X\left(S_{\alpha}(t)\right)$ solves FFPE (1.1). The concept of subordination in terms of a coupled Langevin equation was originally introduced in [7].

FFPE describing subdiffusion in the presence of time-dependent force $F(t)$ was derived in 37] and has the form

$$
\frac{\partial w(x, t)}{\partial t}=\left[-F(t) \frac{\partial}{\partial x}+\frac{\sigma^{2}}{2} \frac{\partial^{2}}{\partial x^{2}}\right]{ }_{0} D_{t}^{1-\alpha} w(x, t),
$$

$w(x, 0)=\delta(x)$. Compared to (1.1), we can observe that the fractional operator ${ }_{0} D_{t}^{1-\alpha}$ in the above equation does not act on the force $F(t)$, so the force is not influenced by the change of time.

Stochastic process corresponding to (1.4) has the form 15]

$$
Y(t)=\int_{0}^{t} F(u) d S_{\alpha}(u)+\sigma B\left(S_{\alpha}(t)\right), \quad t \geq 0,
$$

meaning that the PDF of $Y(t)$ solves (1.4). $Y(t)$ in (1.5) can be equivalently represented in the form of subordination

$$
Y(t)=X\left(S_{\alpha}(t)\right)
$$

where $X$ is given by

$$
d X(t)=F\left(T_{\alpha}(t)\right) d t+\sigma d B(t), \quad X(0)=0,
$$

and $S_{\alpha}$ is the inverse subordinator independent of $B$. Note that in this case processes $X$ and $S_{\alpha}$ are not independent, since $T_{\alpha}$ and its inverse $S_{\alpha}$ are strongly dependent; see formula (1.3).

Further relevant studies of FFPE in the $\alpha$-stable case include: fractional Cauchy problems [6, 23, 32, path properties of fractional diffusion [16, 21, 33], Pearson diffusion [13] and the case of general forces [8,14]. In the recent paper [18] the case of a general space-time-dependent force and diffusion coefficients is considered.

Extension of (1.5) (or equivalently (1.6) ) to the ID case is the following: by $T_{\Psi}(t), t \geq 0$ we denote a subordinator (strictly increasing Lévy process) with Laplace transform [2]

$$
\mathbb{E}\left(e^{-u T_{\Psi}(t)}\right)=e^{-t \Psi(u)} .
$$

Here $\Psi(u)$ is the Laplace exponent given by

$$
\Psi(u)=\lambda u+\int_{0}^{\infty}\left(1-e^{-u x}\right) \nu(d x) .
$$

We assume for simplicity that $\lambda=0$. The measure $\nu(d x)$ is the Lévy measure supported in $(0, \infty)$ satisfying $\int_{(0, \infty)} \min (1, x) \nu(d x)<\infty$. To exclude the case of compound Poisson processes, we will further assume that $\nu((0, \infty))=\infty$. The corresponding first-passage-time process

$$
S_{\Psi}(t)=\inf \left\{\tau>0: T_{\Psi}(\tau)>t\right\}, \quad t \geq 0,
$$

is called an inverse subordinator. Inverse subordinators play an important role in probability theory [2,3], finance [40] and physics [11,39]. Since $\nu((0, \infty))=\infty$, the trajectories of $S_{\Psi}(t)$ are continuous, which is relevant in physical applications. 
Now, to extend (1.5) and (1.6) to the ID case, we replace the inverse stable subordinator $S_{\alpha}$ with $S_{\Psi}$ and obtain

$$
Y(t)=\int_{0}^{t} F(u) d S_{\Psi}(u)+\sigma B\left(S_{\Psi}(t)\right), \quad t \geq 0,
$$

or equivalently

$$
Y(t)=X\left(S_{\Psi}(t)\right)
$$

In this case $X$ is given by

$$
d X(t)=F\left(T_{\Psi}(t)\right) d t+\sigma d B(t), \quad X(0)=0,
$$

where the subordinator $T_{\Psi}$ is independent of Brownian motion $B$. The process $Y$ defined in (1.8) and (1.9) describes subdiffusion with ID waiting times in the presence of time-dependent force $F(t)$ 17, 37. The corresponding fractional FokkerPlanck equation has the form [17, 37]

$$
\frac{\partial w(x, t)}{\partial t}=\left[-F(t) \frac{\partial}{\partial x}+\frac{\sigma^{2}}{2} \frac{\partial^{2}}{\partial x^{2}}\right] \Phi_{t} w(x, t) .
$$

The integro-differential operator $\Phi_{t}$ is defined as

$$
\Phi_{t} f(t)=\frac{d}{d t} \int_{0}^{t} M(t-y) f(y) d y
$$

where $f$ is a sufficiently smooth function. Moreover, the kernel $M(t)$ is defined via its Laplace transform

$$
\mathcal{L}[M](u)=\int_{0}^{\infty} e^{-u t} M(t) d t=\frac{1}{\Psi(u)},
$$

where $\Psi(u)$ is the Laplace exponent of the underlying ID distribution of waiting times.

In addition to the previously mentioned $\alpha$-stable case, other important application examples include: the tempered-stable case [17,22,25,35, which corresponds to $\Psi(u)=(u+\lambda)^{\alpha}-\lambda^{\alpha}$ with $\lambda>0$ and $0<\alpha<1$; the distributed order case [5, 12, 24, which corresponds to $\Psi(u)=\int_{0}^{\infty}\left(1-e^{-u x}\right) \nu(d x)$ with $\nu(t, \infty)=\int_{0}^{1} t^{-\beta} \mu(d \beta)$ (here $\beta \in(0,1)$ and $\mu$ is some distribution supported in $[0,1])$.

Another extension of (1.9) is possible when we add a Lévy noise $d L(t)$ into equation (1.10). For the Lévy process $L(t)$ we have the Lévy-Khintchine formula

$$
\mathbb{E} e^{i u X(t)}=\exp \left[t\left(i b u-\frac{a^{2} u^{2}}{2}+\int_{\mathbb{R} \backslash\{0\}}\left(e^{i u y}-1-i u y \mathbf{1}_{\{|y|<1\}}\right) \mu(d y)\right)\right],
$$

where $b$ is a drift parameter, $a^{2}$ is connected with the Brownian part of $L(t)$ and $\mu$ is the Lévy measure with $\mu(d y)$ being the intensity of jumps of size $y$. Since in our stochastic differential equations the drift and Brownian part already exist, we may assume without loss of generality that $b=a=0$.

The idea of stochastic representation of FFPE has become very popular in a physical society. In a very recent paper [41] the authors found the stochastic representation in the case of tempered-stable subordination with space-time dependent coefficients. In the next section we significantly extend the results from [17, 18, 41]: we consider the general case of space-time-dependent drift and diffusion coefficients, ID waiting times and Lévy noise. Moreover, we analyze the Fokker-Planck equation resulting from adding the Lévy noise $E(t) d L(t)$ to equation (1.10). The general idea 
of the proof of the main theorem is similar to [18; however, we focus our attention on necessary changes coming from the ID subordinator and the Lévy noise. The most important differences are presented in the proof of the main theorem. We also put our effort into delivering all necessary mathematical details that were lacking in the physical paper [18.

\section{MAin RESUlt}

Now, let us look at the following fractional Fokker-Planck equation:

$$
\begin{aligned}
& \frac{\partial}{\partial t} w(x, t)=\left[-\frac{\partial}{\partial x} F(x, t)+\frac{1}{2} \frac{\partial^{2}}{\partial x^{2}} \sigma^{2}(x, t)\right] \Phi_{t} w(x, t) \\
& \quad+\int_{\mathbb{R} \backslash\{0\}}\left(\left.\Phi_{t} w(r, t)\right|_{r=x-E(t) y}-\Phi_{t} w(x, t)+E(t) y \frac{\partial}{\partial x} \Phi_{t} w(x, t) \mathbf{1}_{\{|y|<1\}}\right) \nu(d y),
\end{aligned}
$$

$w(x, 0)=\delta(x)$. It describes the temporal evolution of $w(x, t)-$ PDF of some anomalous diffusion process with ID waiting times, which is subjected to spacetime-dependent force $F(x, t)$ and diffusion $\sigma(x, t)$ and with time-dependent jump coefficient $E(t)$. In the theorem below, which is the main result of the paper, we derive the stochastic process corresponding to (2.1). The result encompasses the whole family of ID waiting times, arbitrary space-time-dependent drift and diffusion coefficients and Lévy jumps with time-dependent jump coefficient. From now on for the left limit process we will use the notation $X^{-}(t)=\lim _{s \rightarrow t^{-}} X(s)$.

Theorem 2.1. Let $T_{\Psi}(\tau)$ be the subordinator with the Laplace exponent $\Psi(u)$ and $S_{\Psi}(t)$ its inverse. Assume that the standard Brownian motion $B(t)$, the Lévy process $L(t)$ with Lévy measure $\mu$ and $T_{\Psi}(\tau)$ are independent. Let $Y(t)$ be the solution of the stochastic equation

$(2.2) d Y(t)=F\left(Y^{-}(t), T_{\Psi}^{-}(t)\right) d t+\sigma\left(Y^{-}(t), T_{\Psi}^{-}(t)\right) d B(t)+E\left(T_{\Psi}^{-}(t)\right) d L(t), t \geq 0$,

$$
Y(0)=0, \quad T_{\Psi}(0)=0,
$$

where the functions $F(x, t), \sigma(x, t), E \in \mathbf{C}^{2}\left(\mathbb{R}^{2}\right)$ satisfy the Lipschitz condition. We assume that the PDF of the process $\left(Y(t), T_{\Psi}(t)\right)-p_{t}(y, z)$ exists and so do $\frac{\partial}{\partial t} p_{t}(y, z), \frac{\partial}{\partial y} p_{t}(y, z)$ and $\frac{\partial^{2}}{\partial y^{2}} p_{t}(y, z)$. Additionally we require that:

$$
\int_{0}^{t_{0}} \int_{0}^{\infty}\left|\frac{\partial}{\partial t} p_{s}(x, t)\right| d s d t<\infty
$$

for each $t_{0}>0$,

$$
\int_{x_{1}}^{x_{2}} \int_{0}^{\infty}\left|\frac{\partial}{\partial x} p_{s}(x, t)\right| d s d x<\infty, \quad \int_{x_{1}}^{x_{2}} \int_{0}^{\infty}\left|\frac{\partial^{2}}{\partial x^{2}} p_{s}(x, t)\right| d s d x<\infty
$$

for each $x_{1}, x_{2} \in \mathbb{R}$ and finally

$\int_{0}^{\infty} \int_{\mathbb{R} \backslash\{0\}}\left|p_{s}(x-E(t) y, t)-p_{s}(x, t)+\frac{\partial}{\partial x}(E(t) y) p_{s}(x, t) \mathbf{1}_{\{|y|<1\}}(y)\right| \nu(d y) d s<\infty$

for each $x \in \mathbb{R}, t>0$. Then the PDF of the process $X(t)=Y^{-}\left(S_{\Psi}(t)\right)$ is the weak solution of FFPE (2.1), that is, (2.1) holds pointwise for $t>0$ with the required initial distribution for $t=0$. 
Remark 2.2. If we impose additional conditions on the coefficients $F, \sigma, E$, the Lévy measures $\mu$ and $\nu$ (of $L(t)$ and $T_{\Psi}(t)$, respectively) namely: $F, \sigma \in C_{b}^{3}\left(\mathbb{R}^{2}\right.$ ), $E \in C_{b}^{3}(\mathbb{R})$ with bounded partial derivatives of the order $1-3, D(y, z) \neq 0$ for all $y, z \in \mathbb{R}$ and $\int_{0}^{\infty} x^{p} \nu(d x)<\infty, \int_{\mathbb{R}}|x|^{p} \mu(d x)<\infty$ for all $p \geq 2$, then the density $p_{t}(y, z)$ exists; see Theorem 2-14 in [4]. Other, less strict non-degeneracy conditions are also discussed there. The regularity assumptions of $p_{t}(y, z)$ are easy to check when $F(x, t)$ and $\sigma(x, t)$ depend only on $x$ and the processes $Y(t)$ and $T_{\Psi}(t)$ are independent. Notice that we do not assume anything about the PDF of $X(t)$.

Proof. In the proof we will essentially extend the techniques used in [8, 15, 18, Equation (2.2) is equivalent to the following system of stochastic equations:

$$
\begin{aligned}
\left(\begin{array}{c}
d Y(t) \\
d Z(t)
\end{array}\right) & =\left(\begin{array}{c}
F\left(Y^{-}(t), Z^{-}(t)\right) \\
0
\end{array}\right) d t+\left(\begin{array}{c}
\sigma\left(Y^{-}(t), Z^{-}(t)\right) \\
0
\end{array}\right) d B(t) \\
& +\left(\begin{array}{c}
E\left(Z^{-}(t)\right) d L(t) \\
d T_{\Psi}(t)
\end{array}\right) .
\end{aligned}
$$

This system is subjected to Brownian and Lévy noise, therefore the infinitesimal generator for the process $(Y(t), Z(t))$ operates on functions $f \in \mathbf{C}_{0}^{2}\left(\mathbb{R}^{2}\right)$ in the following way (see Theorem 6.7.4 in [1]):

$$
\begin{aligned}
A f(y, z) & =F(y, z) \frac{\partial}{\partial y} f(y, z)+\frac{1}{2} \sigma^{2}(y, z) \frac{\partial^{2}}{\partial y^{2}} f(y, z) \\
& +\int_{\mathbb{R} \backslash\{0\}}\left(f(y+E(z) x, z)-f(y, z)-E(z) x \frac{\partial}{\partial y} f(y, z) \mathbf{1}_{\{|x|<1\}}\right) \mu(d x) \\
& +\int_{0}^{\infty}[f(y, z+u)-f(y, z)] \nu(d u),
\end{aligned}
$$

where $\nu$ is the Lévy measure of $Z(t)$ (or equivalently $T_{\Psi}(t)$ ). We have two separate integrals here, because $L(t)$ and $T_{\Psi}(t)$ are assumed to be independent which implies that $\varrho$ - Lévy measure of the two-dimensional process $\left(L(t), T_{\Psi}(t)\right)$ is supported on the axes of $\mathbb{R}^{2}$, that is, $\varrho(A)=\mu\left(\left\{(y, z) \in \mathbb{R}^{2}:(y, 0) \in A\right\}\right)+\nu(\{z \in \mathbb{R}:(0, z) \in A\})$ for $A \in \operatorname{Bor}\left(\mathbb{R}^{2}\right)$. In the first step of the proof we will obtain an FFPE describing the temporal evolution of the density $p_{t}(y, z)$. To do this we take advantage of the fact that

$$
\frac{\partial}{\partial t} p_{t}(y, z)=A^{+} p_{t}(y, z)
$$

Here $A^{+}$is the $L^{2}$ Hermitian adjoint of $A$, meaning that it satisfies the following relation for all test functions $f \in \mathbf{C}_{c}^{\infty}\left(\mathbb{R}^{2}\right)$ :

$$
\int_{\mathbb{R}^{2}} A f(y, z) p_{t}(y, z) d y d z=\int_{\mathbb{R}^{2}} A^{+} p_{t}(y, z) f(y, z) d y d z
$$

If we substitute (2.7) into the above equation, then the left-hand side consists of four summands. The first two summands are dealt with in [18. Now we turn our attention to the third one - connected with an integration with respect to the 
measure $\mu$. Since $f$ has compact support we can apply Fubini's theorem to get

$$
\begin{aligned}
& \int_{\mathbb{R}^{2}} \int_{\mathbb{R} \backslash\{0\}} \\
& \quad\left(f(y+E(z) x, z)-f(y, z)-E(z) x \frac{\partial}{\partial y} f(y, z) \mathbf{1}_{\{|x|<1\}}\right) \mu(d x) p_{t}(y, z) d(y, z) \\
& =\int_{\mathbb{R} \backslash\{0\}} \int_{\mathbb{R}} \int_{\mathbb{R}} \\
& \quad\left(f(y+E(z) x, z)-f(y, z)-E(z) x \frac{\partial}{\partial y} f(y, z) \mathbf{1}_{\{|x|<1\}}\right) p_{t}(y, z) d y d z \mu(d x) .
\end{aligned}
$$

For the inner integral (for each fixed $z$ and $x$ ) after a substitution and using integration by parts we get

$$
\begin{aligned}
& \int_{\mathbb{R}}\left(f(y+E(z) x, z)-f(y, z)-E(z) x \frac{\partial}{\partial y} f(y, z) \mathbf{1}_{\{|x|<1\}}\right) p_{t}(y, z) d y \\
& =\int_{\mathbb{R}} f(y, z)\left(p_{t}(y-E(z) x, z)-p_{t}(y, z)+E(x) x \frac{\partial}{\partial y} p_{t}(y, z) \mathbf{1}_{\{|x|<1\}}\right) d y .
\end{aligned}
$$

Substituting (2.11) back into (2.10) and using Fubini's theorem again we obtain

$$
\begin{aligned}
& \int_{\mathbb{R}^{2}} \int_{\mathbb{R} \backslash\{0\}} \\
& \quad\left(f(y+E(z) x, z)-f(y, z)-E(z) x \frac{\partial}{\partial y} f(y, z) \mathbf{1}_{\{|x|<1\}}\right) \mu(d x) p_{t}(y, z) d(y, z) \\
& =\int_{\mathbb{R}^{2}} f(y, z) \\
& \quad \int_{\mathbb{R} \backslash\{0\}}\left(p_{t}(y-E(z) x, z)-p_{t}(y, z)+E(z) x \frac{\partial}{\partial y} p_{t}(y, z) \mathbf{1}_{\{|x|<1\}}\right) \mu(d x) d(y, z) .
\end{aligned}
$$

Now let us put

$$
G(u)=\nu((u, \infty)) .
$$

Using the general formula for integration by parts (see (21.68) in [9]), we get for the last summand in equation (2.9)

$$
\begin{aligned}
& \int_{0}^{\infty}[f(y, z+u)-f(y, z)] \nu(d u)=-\lim _{u \rightarrow \infty}(f(y, z+u)-f(y, z)) G(u) \\
& +\lim _{u \rightarrow 0}(f(y, z+u)-f(y, z)) G(u)+\int_{0}^{\infty} \frac{\partial}{\partial u} f(y, z+u) G(u) d u .
\end{aligned}
$$

It is easy to see that the first limit vanishes. For the second limit we also have

$$
\lim _{u \rightarrow 0}(f(y, z+u)-f(y, z)) G(u)=\lim _{u \rightarrow 0} \frac{f(y, z+u)-f(y, z)}{u} u G(u)=0,
$$

since $f$ is differentiable and $\lim _{u \rightarrow 0} u G(u)=0$. The last equality follows from the fact that if

$$
\limsup _{u \rightarrow 0} u G(u)>0
$$


then there exist a decreasing sequence $s_{n} \rightarrow 0$ and $d>0$ such that for each $n \in \mathbb{N}$

$$
s_{n} G\left(s_{n}\right)>d \text {. }
$$

We can now find a subsequence $s_{n_{k}}$ satisfying

$$
s_{n_{k}} \nu\left(\left(s_{n_{k+1}}, s_{n_{k}}\right]\right)>\frac{d}{2}
$$

for each $k \in \mathbb{N}$. We reached a contradiction with $\int_{(0, \infty)} \min (1, x) \nu(d x)<\infty$. Thus the limit equals 0 . Taking into account the above calculations, we follow the reasoning from [18]. That gives us for the last summand in (2.9)

$$
\begin{aligned}
& \int_{\mathbb{R}^{2}} \int_{0}^{\infty}[f(y, z+u)-f(y, z)] p_{t}(y, z) \nu(d u) d y d z \\
&=-\int_{-\mathbb{R}^{2}} f(y, w) \frac{\partial}{\partial w} \int_{0}^{w} G(w-z) p_{t}(y, z) d z d y d w .
\end{aligned}
$$

Let us introduce the following operator:

$$
\Theta_{w} g(w)=\int_{0}^{w} G(w-z) g(z) d z .
$$

Laplace transform of its kernel equals

$$
\mathcal{L}[G](u)=\int_{0}^{\infty} e^{-u t} G(t) d t=\int_{0}^{\infty} \int_{(t, \infty)} e^{-u t} \nu(d s) d t=\int_{0}^{\infty} \frac{1}{u}\left(1-e^{-u s}\right) \nu(d s)=\frac{\Psi(u)}{u} .
$$

Combining equations (2.12) and (2.14) we obtain the explicit formula for the adjoint operator $A^{+}$with $p_{t}(y, z)$ in its domain, and from equation (2.8) we finally get

$$
\begin{aligned}
\frac{\partial}{\partial t} p_{t}(y, z)= & -\frac{\partial}{\partial y}\left(F(y, z) p_{t}(y, z)\right)+\frac{\partial^{2}}{\partial y^{2}}\left(\frac{1}{2} \sigma^{2}(y, z) p_{t}(y, z)\right) \\
& +\int_{\mathbb{R} \backslash\{0\}}\left(p_{t}(y-E(z) x, z)-p_{t}(y, z)+E(z) x \frac{\partial}{\partial y} p_{t}(y, z) \mathbf{1}_{\{|x|<1\}}\right) \mu(d x) \\
& -\frac{\partial}{\partial z} \Theta_{z} p_{t}(y, z) .
\end{aligned}
$$

We derived the equation for temporal evolution of $p_{t}(y, z)$ which was our goal in the first step of the proof. Let $w(x, t)$ denote the probability density function of the process $X(t)$. In the next step we will find the relation between the densities $p_{t}(y, z)$ and $w(x, t)$. We start with denoting paths of the analyzed processes as $X(t, \omega)$ and $(Y(t, \omega), Z(t, \omega))$ for each $\omega \in \Omega$. For each fixed interval $I$, similarly as in 8,18 , we define an auxiliary function

$$
H_{t}(s, \omega, u)= \begin{cases}\mathbf{1}_{I}\left(Y^{-}(s, \omega)\right) & \text { if } Z^{-}(s, \omega) \leq t \leq Z^{-}(s, \omega)+u \\ 0 & \text { otherwise, }\end{cases}
$$

and follow, with appropriate changes, the reasoning from the mentioned papers. This includes the observation

$$
\mathbf{1}_{I}(X(t, \omega))=\sum_{s>0} H_{t}(s, \omega, \Delta Z(s, \omega)),
$$

where $\Delta Z(s)=Z(s)-Z^{-}(s)$. In our case the above equation is also valid since we excluded the case of compound Poisson processes and jumping times of $Z(t, \omega)$ 
are dense in $[0, \infty)$ almost surely. The second key equation is the compensation formula (Ch. XII, Proposition (1.10) in 34]

$$
\mathbb{E}\left[\sum_{s>0} H_{t}(s, \omega, \Delta Z(s, \omega))\right]=\mathbb{E}\left[\int_{0}^{\infty} \int_{0}^{\infty} H_{t}(s, \omega, u) \nu(d u) d s\right] .
$$

The difference between our case and 18] is that in the definition of $H_{t}$, instead of $Y(s, \omega)$, we put $Y^{-}(s, \omega)$. Due to the Lévy noise the previous process may no longer be continuous, but $Y^{-}$is still left-continuous and therefore predictable, which is a condition for using the compensation formula. Another condition is that

$$
\sum_{s \in \mathbb{R}^{+}: \Delta Z(s)=0} H_{t}(s, \omega, \Delta Z(s, \omega))=0 \quad \text { almost surely, }
$$

which is also fulfilled. Indeed, assume to the contrary that for $\omega \in W \subset \Omega$ this sum does not vanish and $P(W) \neq 0$. This means that there exists $s>0$ such that $Z^{-}(s, \omega)=t=Z(s, \omega)$. However the paths of the process $Z(s, \omega)$ hit the single point $t$ with probability 0 (see 38 ), which contradicts the assumption. Consequently,

$$
w(x, t)=\int_{0}^{\infty} \Theta_{t} p_{s}(x, t) d s .
$$

Detailed derivation of the above result (with the Riemann-Liouville fractional integral instead of $\left.\Theta_{t}\right)$ is in [18]. Notice that

$$
\frac{d}{d t} \Theta_{t} f(t)=\Theta_{t} \frac{d}{d t} f(t)+f(0) G(t)
$$

for sufficiently smooth $f$. This can be proven calculating Laplace transform: for the left-hand side of (2.22) we get

$$
\mathcal{L}\left[\frac{d}{d t} \Theta_{t} f(t)\right](s)=s \mathcal{L}\left[\Theta_{t} f(t)\right](s)=\Psi(s) \mathcal{L}[f(t)](s),
$$

whereas the right-hand side equals

$$
\mathcal{L}\left[\Theta_{t} \frac{d}{d t} f(t)+f(0) G(t)\right](s)=\frac{\Psi(s)}{s} \mathcal{L}\left[\frac{d}{d t} f(t)\right](s)+f(0) \frac{\Psi(s)}{s}=\Psi(s) \mathcal{L}[f(t)](s) .
$$

Thus

$$
\frac{\partial}{\partial t} \Theta_{t} p_{s}(x, t)=\Theta_{t} \frac{\partial}{\partial t} p_{s}(x, t)+p_{s}(x, 0) G(t)
$$

and we have the following approximation:

$$
\begin{aligned}
\int_{0}^{t_{0}} & \int_{0}^{\infty}\left|\frac{\partial}{\partial t} \Theta_{t} p_{s}(x, t)\right| d s d t \\
& \leq \int_{0}^{t_{0}} \int_{0}^{\infty}\left|\Theta_{t} \frac{\partial}{\partial t} p_{s}(x, t)\right| d s d t+\int_{0}^{t_{0}} \int_{0}^{\infty} p_{s}(x, 0) G(t) d s d t
\end{aligned}
$$

We deal with both integrals separately. For the first one we observe that

$$
\int_{0}^{t_{0}} G(u) d u=\int_{0}^{t_{0}} \int_{(u, \infty)} \mu(d w) d u=\int_{(0, \infty)} \min \left(w, t_{0}\right) \mu(d w)=K<\infty
$$


where $K>0$, and use Fubini's theorem together with the assumption (2.3)

$$
\begin{aligned}
\int_{0}^{t_{0}} & \int_{0}^{\infty}\left|\Theta_{t} \frac{\partial}{\partial t} p_{s}(x, t)\right| d s d t \\
& \leq \int_{0}^{t_{0}} \int_{0}^{\infty} \int_{0}^{t} G(t-u)\left|\frac{\partial}{\partial u} p_{s}(x, u)\right| d u d s d t \\
& \leq K \int_{0}^{t_{0}} \int_{0}^{\infty}\left|\frac{\partial}{\partial u} p_{s}(x, u)\right| d s d u<\infty .
\end{aligned}
$$

For the second integral in (2.23) we obtain

$$
\int_{0}^{t_{0}} \int_{0}^{\infty} p_{s}(x, 0) G(t) d s d t=K \int_{0}^{\infty} p_{s}(x, 0) d s<\infty .
$$

The last inequality is a consequence of Theorem 35.4 in 38. Combining the approximations for both integrals we get

$$
\int_{0}^{t_{0}} \int_{0}^{\infty}\left|\frac{\partial}{\partial t} \Theta_{t} p_{s}(x, t)\right| d s d t<\infty
$$

Therefore after differentiating equation (2.21) with respect to $t$ we can move the derivative inside the integral:

$$
\frac{\partial}{\partial t} w(x, t)=\int_{0}^{\infty} \frac{\partial}{\partial t} \Theta_{t} p_{s}(x, t) d s .
$$

Now, applying equation (2.17), taking into account the facts that $\lim _{s \rightarrow \infty} p_{s}(x, t)=$ 0 and $p_{0}(x, t)=\delta_{(0,0)}(x, t)$ - the two-dimensional Dirac delta, we obtain for $t>0$

$$
\begin{aligned}
\frac{\partial}{\partial t} w(x, t)= & \int_{0}^{\infty}\left[\frac{\partial^{2}}{\partial x^{2}}\left(\frac{1}{2} \sigma^{2}(x, t) p_{s}(x, t)\right)-\frac{\partial}{\partial x}\left(F(x, t) p_{s}(x, t)\right)\right. \\
& +\int_{\mathbb{R} \backslash\{0\}}\left(p_{s}(x-E(t) y, t)-p_{s}(x, t)+E(t) y \frac{\partial}{\partial x} p_{s}(x, t) \mathbf{1}_{\{|y|<1\}}\right) \nu(d y) \\
& \left.-\frac{\partial}{\partial s} p_{s}(x, t)\right] d s \\
= & \frac{\partial^{2}}{\partial x^{2}}\left(\frac{1}{2} \sigma^{2}(x, t) \int_{0}^{\infty} p_{s}(x, t) d s\right)-\frac{\partial}{\partial x}\left(F(x, t) \int_{0}^{\infty} p_{s}(x, t) d s\right) \\
& +\int_{\mathbb{R} \backslash\{0\}}\left[\int_{0}^{\infty} p_{s}(x-E(t) y, t) d s-\int_{0}^{\infty} p_{s}(x, t) d s\right. \\
& +E(t) y \frac{\partial}{\partial x}\left(\int_{0}^{\infty} p_{s}(x, t) d s\right) \mathbf{1}_{\{|y|<1\}]} \nu(d y) .
\end{aligned}
$$

We can interchange the derivative and the integral here because of the assumptions (2.4). Changing the order of integration, $d s$ with $\nu(d y)$, is justified based on Fubini's theorem and the assumption (2.5). Next we apply Fubini-Tonelli theorem again, this time to equation (2.21), obtaining

$$
w(x, t)=\int_{0}^{\infty} \int_{0}^{t} G(t-z) p_{s}(x, z) d z d s=\Theta_{t} \int_{0}^{\infty} p_{s}(x, t) d s,
$$


because the function $G(t-z) p_{s}(x, z)$ is non-negative. Therefore

$$
\int_{0}^{\infty} p_{s}(x, t) d s=\Theta_{t}^{-1} w(x, t)
$$

and

$$
\int_{0}^{\infty} p_{s}(x-E(t) y, t) d s=\left.\left(\Theta_{t}^{-1} w(r, t)\right)\right|_{r=x-E(t) y},
$$

where $\Theta_{t}^{-1}$ is the left-inverse of the operator $\Theta_{t}$ defined in (2.15). It turns out that $\Theta_{t}^{-1}=\Phi_{t}$ (see equation (1.12) $)$. Indeed, one can easily notice that $\Theta_{t}$ is a convolution operator with the kernel $G(u)$ and, similarly, $\Phi_{t}$ is a composition of a derivative operator and a convolution operator with the kernel $M(u)$. Therefore, using equations (2.16) and (1.13) we get

$$
\mathcal{L}\left[\Phi_{t} \Theta_{t} f(t)\right](u)=u \mathcal{L}[f](u) \mathcal{L}[M](u) \mathcal{L}[G](u)=u \mathcal{L}[f](u) \frac{1}{\Psi(u)} \frac{\Psi(u)}{u}=\mathcal{L}[f](u)
$$

for a sufficiently smooth function $f$, which implies that $\Phi_{t} \Theta_{t} f(t)=f(t)$. Consequently, from equations (2.29) and (2.31) we obtain the desired result.

The proof cannot be easily extended to the case where the jump coefficient $\mathrm{E}$ is space dependent. In such situation the operator $A^{+}$does not exist.

Remark 2.3. The above theorem can be used to approximate solutions of FFPE (2.1) using Monte Carlo methods based on realizations of the process $X(t)$. Indeed, to simulate trajectories of $X(t)$, one only needs to simulate the process $Y(t)$ (using the standard Euler scheme [10]) and the inverse subordinator $S_{\Psi}(t)[15,17$.

It also opens the possibility of analyzing fractional Cauchy problems [13, 23, 25] in the general setting of ID subordinators and arbitrary space-time-dependent drift and diffusion coefficients.

\section{ACKNOWLEDGEMENT}

This research was partially supported by NCN Maestro grant no. 2012/06/A/ ST1/00258.

\section{REFERENCES}

[1] David Applebaum, Lévy processes and stochastic calculus, Cambridge Studies in Advanced Mathematics, vol. 93, Cambridge University Press, Cambridge, 2004. MR 2072890 (2005h:60003)

[2] Jean Bertoin, Lévy processes, Cambridge Tracts in Mathematics, vol. 121, Cambridge University Press, Cambridge, 1996. MR1406564 (98e:60117)

[3] Jean Bertoin, Subordinators: examples and applications, Lectures on probability theory and statistics (Saint-Flour, 1997), Lecture Notes in Math., vol. 1717, Springer, Berlin, 1999, pp. 191, DOI 10.1007/978-3-540-48115-7_1. MR.1746300(2002a:60001)

[4] Klaus Bichteler, Jean-Bernard Gravereaux, and Jean Jacod, Malliavin calculus for processes with jumps, Stochastics Monographs, vol. 2, Gordon and Breach Science Publishers, New York, 1987. MR 1008471 (90h:60056)

[5] A.V. Chechkin, R. Gorenfo and I.M. Sokolov, Retarding subdiffusion and accelerating superdiffusion governed by distributed order fractional diffusion equations, Phys. Rev. E, 66 (2002), 1-7.

[6] Zhen-Qing Chen, Mark M. Meerschaert, and Erkan Nane, Space-time fractional diffusion on bounded domains, J. Math. Anal. Appl. 393 (2012), no. 2, 479-488, DOI 10.1016/j.jmaa.2012.04.032. MR.2921690 
[7] H. C. Fogedby, Langevin equations for continuous time Lévy flights, Phys. Rev. E 50 (1994), 1657-1701.

[8] B. I. Henry, T. A. M. Langlands, and P. Straka, Fractional Fokker-Planck equations for subdiffusion with space- and time-dependent forces, Phys. Rev. Lett., 105 (2010), 170602.

[9] Edwin Hewitt and Karl Stromberg, Real and abstract analysis, Springer-Verlag, New YorkHeidelberg, 1975. A modern treatment of the theory of functions of a real variable; Third printing; Graduate Texts in Mathematics, No. 25. MR0367121 (51 \#3363)

[10] Aleksander Janicki and Aleksander Weron, Simulation and chaotic behavior of $\alpha$-stable stochastic processes, Monographs and Textbooks in Pure and Applied Mathematics, vol. 178, Marcel Dekker, Inc., New York, 1994. MR1306279 (96g:60026)

[11] A. Jurlewicz, K. Weron, and M. Teuerle, Generalized Mittag-Leffler relaxation: Clusteringjump continuous-time random walk approach, Phys. Rev. E, 78, (2008), 011103.

[12] Anatoly N. Kochubei, Distributed order calculus and equations of ultraslow diffusion, J. Math. Anal. Appl. 340 (2008), no. 1, 252-281, DOI 10.1016/j.jmaa.2007.08.024. MR2376152 (2009i:35177)

[13] Nikolai N. Leonenko, Mark M. Meerschaert, and Alla Sikorskii, Fractional Pearson diffusions, J. Math. Anal. Appl. 403 (2013), no. 2, 532-546, DOI 10.1016/j.jmaa.2013.02.046. MR.3037487

[14] Longjin Lv, Weiyuan Qiu, and Fuyao Ren, Fractional Fokker-Planck equation with space and time dependent drift and diffusion, J. Stat. Phys. 149 (2012), no. 4, 619-628, DOI 10.1007/s10955-012-0618-3. MR2998593

[15] Marcin Magdziarz, Stochastic representation of subdiffusion processes with timedependent drift, Stochastic Process. Appl. 119 (2009), no. 10, 3238-3252, DOI 10.1016/j.spa.2009.05.006. MR2568272(2011d:60128)

[16] Marcin Magdziarz, Path properties of subdiffusion-a martingale approach, Stoch. Models 26 (2010), no. 2, 256-271, DOI 10.1080/15326341003756379. MR2739351 (2011k:60129)

[17] Marcin Magdziarz, Langevin picture of subdiffusion with infinitely divisible waiting times, J. Stat. Phys. 135 (2009), no. 4, 763-772, DOI 10.1007/s10955-009-9751-z. MR2546742 (2011a:82073)

[18] Marcin Magdziarz, Janusz Gajda, and Tomasz Zorawik, Comment on fractional FokkerPlanck equation with space and time dependent drift and diffusion, J. Stat. Phys. 154 (2014), no. 5, 1241-1250, DOI 10.1007/s10955-014-0919-9. MR3176407

[19] M. Magdziarz, A. Weron, and K. Weron, Fractional Fokker-Planck dynamics: Stochastic representation and computer simulation, Phys. Rev. E, 75 (2007), 016708.

[20] Mark M. Meerschaert, David A. Benson, Hans-Peter Scheffler, and Boris Baeumer, Stochastic solution of space-time fractional diffusion equations, Phys. Rev. E (3) 65 (2002), no. 4, 041103, 4, DOI 10.1103/PhysRevE.65.041103. MR1917983 (2003d:60165)

[21] Mark M. Meerschaert, Erkan Nane, and Yimin Xiao, Large deviations for local time fractional Brownian motion and applications, J. Math. Anal. Appl. 346 (2008), no. 2, 432-445, DOI 10.1016/j.jmaa.2008.05.087. MR.2431539(2009m:60065)

[22] M. M. Meerschaert, Y. Zhang, and B. Baeumer, Tempered anomalous diffusion in heterogeneous systems, Geophys. Res. Lett., 35 (2008), L17403.

[23] Mark M. Meerschaert, Erkan Nane, and P. Vellaisamy, Fractional Cauchy problems on bounded domains, Ann. Probab. 37 (2009), no. 3, 979-1007, DOI 10.1214/08-AOP426. MR2537547(2010h:60121)

[24] Mark M. Meerschaert, Erkan Nane, and P. Vellaisamy, Distributed-order fractional diffusions on bounded domains, J. Math. Anal. Appl. 379 (2011), no. 1, 216-228, DOI 10.1016/j.jmaa.2010.12.056. MR2776466 (2012e:35263)

[25] Mark M. Meerschaert, Erkan Nane, and P. Vellaisamy, Transient anomalous sub-diffusion on bounded domains, Proc. Amer. Math. Soc. 141 (2013), no. 2, 699-710, DOI 10.1090/S00029939-2012-11362-0. MR 2996975

[26] Mark M. Meerschaert and Hans-Peter Scheffler, Limit theorems for continuous-time random walks with infinite mean waiting times, J. Appl. Probab. 41 (2004), no. 3, 623-638. MR2074812(2005f:60105)

[27] Mark M. Meerschaert and Alla Sikorskii, Stochastic models for fractional calculus, de Gruyter Studies in Mathematics, vol. 43, Walter de Gruyter \& Co., Berlin, 2012. MR2884383 
[28] Ralf Metzler, Generalized Chapman-Kolmogorov equation: a unifying approach to the description of anomalous transport in external fields, Phys. Rev. E (3) 62 (2000), no. 5, 6233-6245, DOI 10.1103/PhysRevE.62.6233. MR.1796441 (2001i:82050)

[29] R. Metzler, E. Barkai, and J. Klafter, Anomalous diffusion and relaxation close to thermal equilibrium: A fractional Fokker-Planck equation approach, Phys. Rev. Lett., 82 (1999), 3563-3567.

[30] R. Metzler, E. Barkai, and J. Klafter, Deriving fractional Fokker-Planck equations from a generalised master equation, Europhys. Lett. 46 (1999), no. 4, 431-436, DOI 10.1209/epl/i199900279-7. MR1687557 (2000a:82052)

[31] Ralf Metzler and Joseph Klafter, The random walk's guide to anomalous diffusion: a fractional dynamics approach, Phys. Rep. 339 (2000), no. 1, 77, DOI 10.1016/S03701573(00)00070-3. MR 1809268(2001k:82082)

[32] Jebessa B. Mijena and Erkan Nane, Strong analytic solutions of fractional Cauchy problems, Proc. Amer. Math. Soc. 142 (2014), no. 5, 1717-1731, DOI 10.1090/S0002-9939-2014-11905-8. MR.3168478

[33] Erkan Nane, Laws of the iterated logarithm for a class of iterated processes, Statist. Probab. Lett. 79 (2009), no. 16, 1744-1751, DOI 10.1016/j.spl.2009.04.013. MR.2566748(2010i:60100)

[34] Daniel Revuz and Marc Yor, Continuous martingales and Brownian motion, 3rd ed., Grundlehren der Mathematischen Wissenschaften [Fundamental Principles of Mathematical Sciences], vol. 293, Springer-Verlag, Berlin, 1999. MR.1725357 (2000h:60050)

[35] Jan Rosiński, Tempering stable processes, Stochastic Process. Appl. 117 (2007), no. 6, 677707, DOI 10.1016/j.spa.2006.10.003. MR2327834 (2008g:60146)

[36] S. G. Samko, A. A. Kilbas, and D. I. Maritchev, Integrals and Derivatives of the Fractional Order and Some of Their Applications, Gordon and Breach, Amsterdam, 1993.

[37] I.M. Sokolov and J. Klafter, Field-induced dispersion in subdiffusion, Phys. Rev. Lett., 97 (2006), 140602.

[38] Ken-iti Sato, Lévy processes and infinitely divisible distributions, Cambridge Studies in Advanced Mathematics, vol. 68, Cambridge University Press, Cambridge, 1999. Translated from the 1990 Japanese original; Revised by the author. MR 1739520 (2003b:60064)

[39] Aleksander Stanislavsky, Karina Weron, and Aleksander Weron, Diffusion and relaxation controlled by tempered $\alpha$-stable processes, Phys. Rev. E (3) 78 (2008), no. 5, 051106, 6, DOI 10.1103/PhysRevE.78.051106. MR2551366 (2010i:82145)

[40] Matthias Winkel, Electronic foreign-exchange markets and passage events of independent subordinators, J. Appl. Probab. 42 (2005), no. 1, 138-152. MR2144899(2006b:60102)

[41] Yun-Xiu Zhang, Hui Gu, and Jin-Rong Liang, Equivalence of Subordinated Processes with Tempered $\alpha$-Stable Waiting Times and Fractional Fokker-Planck Equations in Space and Time Dependent Fields, J. Stat. Phys. 159 (2015), no. 6, 1495-1503, DOI 10.1007/s10955014-1184-7. MR 3350380

Hugo Steinhaus Center, Department of Mathematics, Wroclaw University of TechNOLOGY, 50-370 Wroclaw, Poland

E-mail address: marcin.magdziarz@pwr.wroc.pl

Hugo Steinhaus Center, Department of Mathematics, Wroclaw University of TechNOLOGY, 50-370 Wroclaw, Poland 